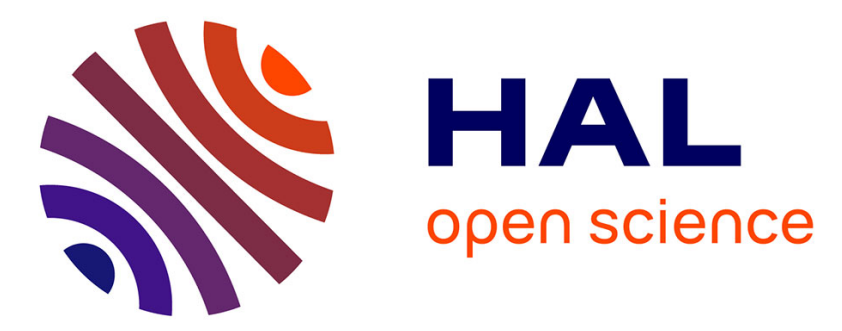

\title{
A MAS-Based Approach for POI Group Recommendation in LBSN
}

Silvia Schiaffino, Daniela Godoy, J. Andrés Díaz Pace, Yves Demazeau

\section{To cite this version:}

Silvia Schiaffino, Daniela Godoy, J. Andrés Díaz Pace, Yves Demazeau. A MAS-Based Approach for POI Group Recommendation in LBSN. 18th International Conference on Practical Applications of Agents and Multi-Agent Systems, PAAMS'20, Oct 2020, L'Aquila, Italy. pp.238-250, 10.1007/978-3030-49778-1_19. hal-02964868

\section{HAL Id: hal-02964868 https://hal.science/hal-02964868}

Submitted on 10 Dec 2020

HAL is a multi-disciplinary open access archive for the deposit and dissemination of scientific research documents, whether they are published or not. The documents may come from teaching and research institutions in France or abroad, or from public or private research centers.
L'archive ouverte pluridisciplinaire HAL, est destinée au dépôt et à la diffusion de documents scientifiques de niveau recherche, publiés ou non, émanant des établissements d'enseignement et de recherche français ou étrangers, des laboratoires publics ou privés. 


\title{
A MAS-based approach for POI group recommendation in LBSN
}

\author{
Silvia Schiaffino ${ }^{1}$, Daniela Godoy ${ }^{1}$, J. Andrés Diaz Pace ${ }^{1}$, and Yves Demazeau ${ }^{2}$ \\ 1 ISISTAN (UNCPBA-CONICET), Tandil, Argentina. \\ ${ }^{2}$ Laboratoire d'Informatique de Grenoble (CNRS), Grenoble, France.
}

\begin{abstract}
Location-based recommender systems (LBRS) suggest friends, events, and places considering information about geographical locations. These recommendations can be made to individuals but also to groups of users, which implies satisfying the group as a whole. In this work, we analyze different alternatives for POI group recommendations based on a multi-agent system consisting of negotiating agents that represent a group of users. The results obtained thus far indicate that our multi-agent approach outperforms traditional aggregation approaches, and that the usage of LBSN information helps to improve both the quality of the recommendations and the efficiency of the recommendation process.
\end{abstract}

Keywords: group recommender systems; location-based social networks; multi-agent systems; negotiation

\section{Introduction}

Location-based social networks (LBSN), like Foursquare or Yelp, take advantage of the advances in communication technologies to enable users to share their geographical location, look for interesting places (POIs, Points Of Interest) and share content and opinions about these places. In this context, location-based recommender systems (LBRS) generally make recommendations to individual users. However, going to a restaurant or visiting a museum are activities that are usually done in groups. Group recommender systems can provide recommendations to a group of users trying to fulfill the expectations of the group as a whole and to satisfy the individual preferences of all group members [10].

Most approaches for group recommendation make use of different aggregation techniques: (i) generating a group profile that combines individual user profiles (profile aggregation), (ii) aggregating recommendations obtained for each group member, such as in ranking aggregation (recommendation aggregation), or (iii) aggregating individual ratings using approaches such as minimizing misery or maximizing average satisfaction (preference aggregation). Although these approaches have been widely applied, they still present some shortcomings. On one hand, some aggregation techniques, such as least misery and average, can generate recommendations that might not reflect the group preferences correctly. On the other hand, the decision-making process of the group and the group dynamics [7] (such as users' influence or trust relationships) are not comprehended by the aggregation techniques [10] (ch. 22). We believe that these aspects are 
important in domains like POI recommendation, in which group members generally discuss and analyze their options for achieving a consensus. Thus, taking the group dynamics into account can help to further personalize recommendations.

Modeling a LBRS as a multi-agent system (MAS) is a more adequate solution, as a negotiation among cooperative agents could replace aggregation techniques, thus helping to overcome their limitations. Along this line, in [15] we proposed a MAS-based approach for group recommendation called MAGReS (Multi-Agent Group Recommender System), which relies on the agents to select those individual recommendations that will be part of the group recommendations. In MAGReS, which was evaluated in the movies domain, each user in a group is represented by a personal agent, which knows the user's preferences and acts on her behalf when trying to agree on items (POIs) with other agents. In order to reach agreements, the agents engage in a cooperative negotiation process.

In the POI domain, group LBRS can leverage on an additional layer of information provided by the LBSN, which includes users' social and geographical relationships, in order to generate better POIs suggestions for the group. In this paper we analyze different alternatives for POI group recommendation and compare our MAGReS approach to two traditional aggregation approaches: aggregation of preferences/ratings, and aggregation of recommendations. Additionally, to determine the usefulness of the LBSN information, we study how the geographical relationships of group members influence the neighbors selection process. The experiments were conducted using the Yelp ${ }^{3}$ dataset. The results obtained thus far suggest that: i) our MAS-based approach outperforms traditional aggregation approaches, and ii) the usage of LBSN information helps to improve the quality of the group recommendations while also making the neighbors selection process more efficient.

The rest of the article is organized as follows. In Section 2 we describe related works. In Section 3 we introduce the MAGReS approach for group recommendation. In Section 4 we present an evaluation of our approach against existing approaches. Finally, in Section 5 we give the conclusions and outline future work.

\section{Related Work}

Most group recommendation strategies are based on the aggregation of recommendations, preferences or profiles [10]. Adaptations of the recommendation aggregation functions are proposed in $[16,18,1]$. In [16] the authors present a group POI recommendation method (GA-GPOI) that combines users' gregariousness, i.e. the degree of association between users in a community, activity, and sign-in times. Zhu et al. [18] consider the rationality of the location, based on distance and the intra-group influence when making group decisions about POIs. The group decision strategy aims to reach a consensus on the POIs based on a function that aggregates the recommendation results taking into account group relevance and group disagreement. Experiments reported in [1] with a hybrid RS that combines the group geographical preferences, category and location features, and group check-ins for generating group suggestions, yield to the

\footnotetext{
${ }^{3}$ http://www . yelp.com/dataset/challenge
} 
conclusions that models with categorical or geographical information performed better than those not using these features. Also, the combination of individual recommendations with aggregation functions underperforms the group models.

Preference aggregation implies to obtain group models to generate predictions. An example is the SPTW-GRM model [9], which creates a common group profile including the common location categories of their members. Thus, the location category with the higher repetition in the group profile shows the users' interests towards such location type and it has more impact on POI recommendations than those with lower repetition. The CGAR approach uses a hierarchical Bayesian model to recommend activities to groups [8]. CGAR uses topic models to mine activities from location information and group preference from user-group membership information, and then a matrix factorization to match the latent feature space of a group to the latent features of locations.

Finally, a profile aggregation approach is presented in [5]. The structure of groups visiting POIs is used for identifying which groups are likely to visit a place, assuming that the group size and structure affect the POI selection (e.g., some places are visited by large groups and others are preferred by small groups.

\section{POI Group Recommendation}

Our proposed approach, called MAGReS (Multi-Agent Group Recommender System), consists of a MAS in which each agent represents a group member. It was initially developed for group recommendation of movies [15], but it can be extended to other domains like POIs. Instead of using traditional aggregation techniques (such as average or least misery), MAGReS relies on a MAS for selecting those individual recommendations that will be part of the group recommendations.

In MAGReS, each agent has access to a user profile that contains the user's preferences regarding POIs. Each agent is capable of (i) predicting the rating the user would assign to a POI not yet rated, and (ii) generating a ranking of potentially interesting POIs for the user. Initially, the user's preferences are the ratings assigned by the user to the POIs she rated in the past. Also, the agent keeps information about the user's social network and geographical information about the places rated or marked as "check-ins". A general overview of our approach is shown in Figure 1. At the core of MAGReS is a negotiation process in which User Agents try to reach a consensus on the most satisfying items for the group. Although several negotiation protocols are available, only a few of them address two important properties for us, namely: (i) mimic the negotiation process followed by humans, and (ii) be suitable for multi-lateral negotiation. On this basis, we chose the Monotonic Concession Protocol (MCP) [3] for the approach. In MCP, a set of cooperative agents negotiate over proposals in order to reach consensus over those proposals to guide the negotiation.

Formalizing the problem, let $A=\left\{a g_{1}, a g_{2}, \ldots, a g_{n}\right\}$ be a finite set of $N$ cooperative agents, and let $X=\left\{x_{1}, x_{2}, \ldots x_{m}\right\}$ be a finite set of potential agreements or proposals, each one of them containing a POI (item) that can be recommended to one of the agents. Each agent $a g_{i} \in A$ has a utility function $U_{i}: X \rightarrow[0,1]$ that maps proposals to their satisfaction value. In our approach, each agent $a g_{i}$ 


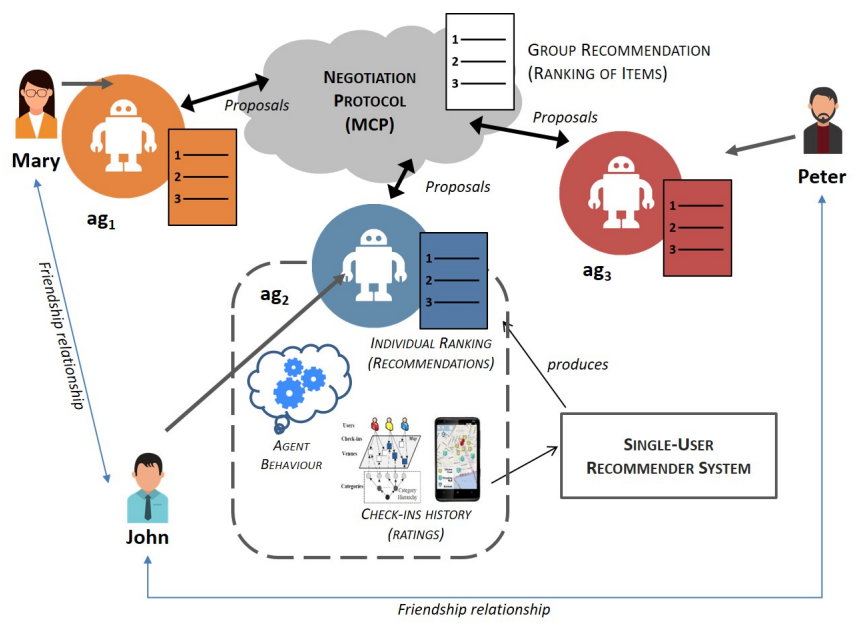

Figure 1: MAGReS approach for recommending POIs to groups

relies on a single-user recommender system $S U R_{i}$, to generate a ranking containing the POIs it can propose. The ranking is sorted in descendant order according to the utility value of the item, and all the candidate proposals with a utility value lower than a certain threshold are discarded. Thus, the set $X$ can be seen as the union of the rankings produced for all the agents, plus a special agreement called conflict deal, which yields utility 0 for all the agents and will be chosen as the worst possible outcome (when no agreement is possible).

For example, in the domain of LBSNs let us assume that a group of three friends wants to go to a restaurant together and there is a set of $P$ possible restaurants to be chosen. According to MAGReS, each user is assigned to a personal agent that handles her profile. For simplicity, a user profile includes only ratings over (a subset of) the possible restaurants. A user rating $r t_{i}($ item $)$ is a value (in the range $[0,1]$ where 0 means dissatisfaction and 1 means high satisfaction) assigned by the user $i$ to the given POI or restaurant. Additionally, the utility/satisfaction function yield by each agent $a g_{i} \in A$ is defined as follows:

$$
U_{i}\left(x_{j}\right)= \begin{cases}r t_{i}\left(x_{j}\right) & \text { if } x_{j} \in R_{i} \\ S U R_{i}\left(x_{j}\right) & \text { if } x_{j} \notin R_{i}\end{cases}
$$

where $R_{i}$ is the list of items rated by user $i$ (or $a g_{i}$ ) and $S U R_{i}\left(x_{j}\right)$ is the rating predicted (in case item $x_{j}$ was not rated by the user $i$, i.e. $x_{j}$ is not in $R_{i}$ ) by the $S U R_{i}$ internally used by $a g_{i}$ for generating its list of candidate proposals.

In this context, let us consider the following (initial) situation: $a g_{1}$ manages ratings $<r t_{1}(P O I 1)=0.6, r t_{1}(P O I 2)=0.8>$ for user $\# 1, a g_{2}$ manages $<r t_{2}(P O I 1)=0.4, r t_{3}(P O I 3)=0.6>$ for user $\# 2$, and $a g_{3}$ manages $<$ $r t_{3}(P O I 2)=0.2, r t_{3}(P O I 3)=0.8>$ for user $\# 3$. Then, $A=\left\{a g_{1}, a g_{2}, a g_{3}\right\}$ is the MAS that carries out the negotiation for the "best" POI or restaurant to 
visit (i.e., the one that will satisfy all the agents). Since these users are friends, we also asssume they are related in a social network.

\subsection{The Monotonic Concession Protocol (MCP)}

MCP [3] is a multi-lateral negotiation protocol for cooperative agents. It is intended to mimic, in a simplified way, the negotiation process carried out by humans when trying to make agreements on topics. The agents engage in negotiation rounds, each agent making proposals of items that need to be assessed by the other agents, until an agreement is reached or the negotiation finishes with a conflict. The agents abide by predefined rules that specify the range of "legal" moves available at each agent at any stage of the process. These rules are related to: (i) the agreement criterion, (ii) which agent makes the next concession (after a round with no agreement), and (iii) how much an agent should concede. The protocol assumes that an agent cannot influence the negotiation position of others, and it assigns quantitative utilities to proposals. For more details about the MCP instantiation for MAGReS, please refer to [15].

At the beginning, each agent makes an initial proposal according to a predefined strategy. For example, if the agent employs an Egocentric concession strategy, it might propose its "favorite" or top-ranked item. After that, the initial proposals of all agents are exchanged in order to determine if an agreement over one of those proposals can be reached. The notion of agreement or deal is defined in terms of the utility of a given proposal. There is an agreement if one agent makes a proposal that is at least as good for any other agent as their own current proposals. In case of a deal, the proposal that satisfies all the agents is returned as the group recommendation. But, if no agreement can be reached, one (or more) of the agents must concede. A concession means that an agent looks for an inferior proposal in terms of its own utility, with the hope of reaching a deal. A concession decision rule decides wich agent should make a concession, and which proposal should be proposed is determined by a concession strategy. If none of the agents can concede, the process finishes with no-agreement (the conflict deal is returned). One way for selecting the agent(s) that must concede is to apply the Zeuthen strategy [17], based on the concept of willingness to risk conflict (WRC).

Various strategies are discussed in the literature for deciding on the item the conceding agent(s) should propose in the next negotiation round [3]. Some possible strategies are: Strong concession (the proposal is strictly better for each of the other agents), Weak concession (the proposal is better for at least one of the other agents), Utilitarian concession (the proposal increases the sum of utilities of the other agents), Egalitarian Concession (the minimum utility amongst the other agents increases), Nash Concession (the product of utilities of the other agents increases), and Desires Distance (DD) that we proposed in [15] to mitigate the problems of the Nash and Utilitarian strategies. DD attempts to measure how far a candidate proposal is with respect to the desires of the others.

Coming back to our example, let us suppose that the initial proposals of $a g_{1}$, $a g_{2}$ and $a g_{3}$ are POI3, POI2 and POI1 respectively (see Figure 2a) and the agents follow the DD concession strategy. According to the multilateral agree- 


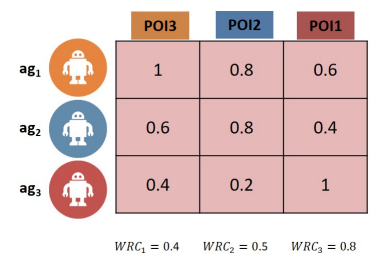

(a) Round 1

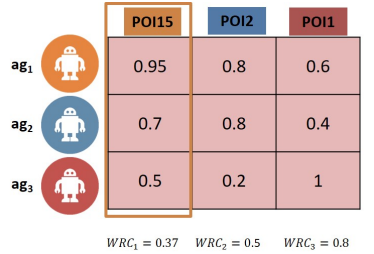

(b) Round 2

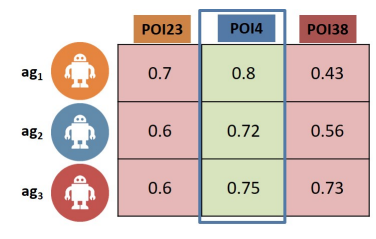

(c) Round $k$

Figure 2: MCP negotiation example over POIs

ment criterion, there is no agreement as none of the proposals satisfies all the agents, and thus one agent must make a concession. According to the concession decision rule, $a g_{1}$ must concede as she has the lowest WRC value. At the beginning of Round 2, $a g_{1}$ proposes POI15, which has a $d d_{\text {value }}$ lower than that of her previous proposal $\left(d d_{\text {value }}(P O I 3)=0.8\right.$ vs. $d d_{\text {value }}(P O I 15)=0.6$. Once again, there is no agreement and one agent must make a concession. The process repeats until reaching Round $k$, in which $a g_{2}$ proposes POI4. As POI14 satisfies all the agents, it is successfully is selected as the group recommendation.

\subsection{Considering information from the LBSN}

Given the variety of data contained in LBSNs, there is a need for developing recommender systems that take advantage of different data sources to enhance recommendations. For example, the traditional collaborative filtering (CF) method, which relates users to items through ratings or opinions provided by users, could be applied straightforwardly to build LBRS. However, CF considers neither the possible friendship relationships nor the geo-localization dimension. In [11], we proposed strategies for including LBSN information when recommending POIs to individual users in a CF context. User-based CF approaches recommend items (e.g. POIs) based on similar users' (called neighbors) preferences. Since userbased CF trusts neighbors as information sources, the quality of recommendations depends directly on the ability to select those neighbors. Our hypothesis was that LBSNs provide rich information for establishing relationships beyond similarity, which enhances the selection of potential neighbors and thus improves the estimation of preferences during the recommendation process.

From experiments using the Foursquare dataset, we concluded that the relationships among users and the use of geo-localization data (among other LSBN elements) allow LBRS to select users that are potentially useful for prediction, particularly in small neighborhoods (up to 100 users). The best performing strategy for selecting neighbors was to choose users from those that share visited places with the target user. This happened because users that tend to visit the same places usually have similar tastes. Notably, this strategy not only reduced the prediction error but also involved the evaluation of fewer users in the prediction step of the $\mathrm{CF}$ approach. These findings led us to include LBSN information in MAGReS to improve the recommendations of POIs to groups. 


\section{Experimental Evaluation}

This section reports on the experiments carried out to evaluate our proposal for POI group recommendation. We used a dataset corresponding to the Yelp Dataset Challenge. Yelp is a LBSN based on the users' check-ins and the dataset includes information about: businesses, reviews, users, friendship relationships and tips. For the purpose of the experiments we used check-ins for the Arizona state (US). Also, we only considered users having at least 9 check-ins (or ratings). We compared the recommendations resulting from traditional group recommendation techniques against those produced by agent negotiation. As baseline for this comparison, we implemented two RS based on traditional approaches from the literature: i) TRADGRec-PA, a GRS that uses preference aggregation [10] (ch. 22); and ii) TRADGRec-RA, a GRS that relies on aggregation of recommendations produced for each group member. This baseline is based on [4](ch. 2), which generates a recommendation containing $k$ items for each group member and then combines them into a single recommendation. We also implemented a variant of MCP, known as the One-Step protocol [12] in which all the negotiations occur in one single round. The agents simply interchange their proposals (one proposal each) and seek for an "agreement", with no concession.

\subsection{Evaluation metrics and Setup}

The goal of the proposed approach is not only to increase group satisfaction but also to make group members uniformly satisfied. Thus, the effectiveness of the recommendations was measured in terms of three satisfaction metrics that can be computed both at the item and recommendation (i.e., a list of items recommended) levels. In all the metrics, the term $S_{i}\left(x_{j}\right)$ represents the satisfaction level of the group member $u_{i}$ over the item $x_{j}$, and it is computed as the rating for the pair $\left\langle u_{i}, x_{j}>\right.$ predicted by the SUR (if $u_{i}$ has rated $x_{j}$ with a rating $r_{u_{i}, x_{j}}$ in the past, then $\left.S_{i}\left(x_{j}\right)=r_{u_{i}, x_{j}}\right)$. The prediction is computed according to the Equation 2, and also according to the similarity metric chosen for the experiment.

$$
r_{u_{i}, x_{j}}=r_{u_{i}}^{-}+\frac{\sum\left(r_{u_{k}, x_{j}}-r_{u_{k}}^{-}\right) \times \operatorname{Similarity}\left(u_{i}, u_{k}\right)}{\sum\left|\operatorname{Similarity}\left(u_{i}, u_{k}\right)\right|}
$$

Group Satisfaction (GS): measures the satisfaction of the group with respect to an item (or a list of items) being recommended. The satisfaction is equivalent to the estimated preference (or rating) of the user/group for the item.

- item level: the group satisfaction for an item $x_{j}$ is computed as explained in Equation 3, where $n$ is the number of group members $(|g|)$ in the group $(g)$ and $S_{i}\left(x_{j}\right)$ is the satisfaction of group member $u_{i}$ over item $x_{j}$.

$$
G S\left(x_{j}\right)=S_{g}\left(x_{j}\right)=\frac{\sum_{i=1}^{n} S_{i}\left(x_{j}\right)}{n}
$$

- recommendation level: the GS of a recommendation $r$ consisting of $k$ items $\left(r=<x_{1}, \ldots, x_{k}>\right)$ is computed as the average of the GS of each item in $r$. 
Members Satisfaction Dispersion (MSD): assesses how uniformly the group members are satisfied by either a single item $x_{j}$ or a recommendation $r$. The lower the MSD is the more uniformly satisfied the group members will be.

- item level: as it can be seen in Equation 4, the MSD for an item $x_{j}$ is computed as the standard deviation of the group members satisfaction.

$$
M S D\left(x_{j}\right)=\sqrt{\frac{\sum_{i=1}^{n}\left(S_{i}\left(x_{j}\right)-S_{g}\left(x_{j}\right)\right)^{2}}{n}}
$$

- recommendation level: the MSD for a recommendation $r$ that consists of $k$ items is computed as the average of the MSD for each item in $r$.

Fairness: metric [14] for evaluating a recommendation of an item $\left(x_{j}\right)$ to a group is defined as the percentage of group members satisfied by the recommendation (Equation 5). To determine which users are satisfied, a threshold th is set to 3.5 stars (out of 5 stars, the equivalent to 0.7 out of 1 ) and any group member with a satisfaction value above that threshold is considered satisfied. We kept th $=0.7$ and extended this metric for a recommendation $r$ of $k$ items. The fairness of a recommendation $r$ of $k$ items is the average of the fairness of the items.

$$
\text { fairness }\left(g, x_{j}\right)=\frac{\left|\bigcup_{u_{i} \in g}: S_{i}\left(x_{j}\right)>t h\right|}{n}
$$

The objectives of the experiments were two-fold: i) to compare the recommendations generated by MAGReS, TRADGRec-PA and TRADGRec-RA in the POI domain; and ii) to study how the usage of LBSN information can affect the recommendations generated by MAGReS and the baselines. The execution of the experiments involved 6 steps, for each approach, as follows: 1) we randomly generate $n$ groups for the test, in which the group members have to be direct friends in the social network; 2) we determine the number of $(k)$ items to be recommended; 3) we configure the environment by: (a) selecting the SUR and neighborhood selection strategies, and (b) parameterizing each approach; 4) we run each approach to generate a group recommendation containing $k$ items for each of the $n$ groups involved in the test; 5 ) we evaluate the recommendations via the GS, MSD and fairness metrics; 6 ) we compare the approaches by computing the average and standard deviation of the metrics.

In total, we create 45 groups of 3,4 and 5 people ( 15 groups of each size). The amount of recommendations $(k)$ is set to 10 , which is a common parameter in the literature (top-10) $[4,6]$. The SUR used by the 3 approaches is the userbased CF recommender implemented on the Mahout framework ${ }^{4}$ along with the Pearson (weighted) similarity metric. With regard to the neighborhood selection strategies, we analyze two alternatives: NearestN (NN) and NearestNUserZone (NNUZ). NN is available in Mahout framework and it does not use LBSN information, while NNUZ [11] uses geolocation information to select the neighbors of a user. This strategy works by first computing the "movement area" (i.e., the

\footnotetext{
${ }^{4}$ http://mahout.apache.org/
} 

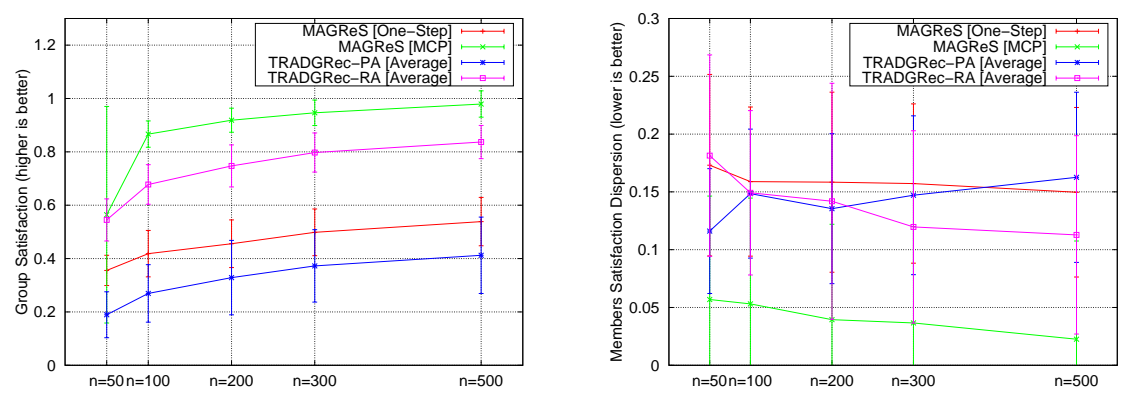

(a) Average GS per approach and neighbor-(b) Average MSD per approach and neighhood size borhood size

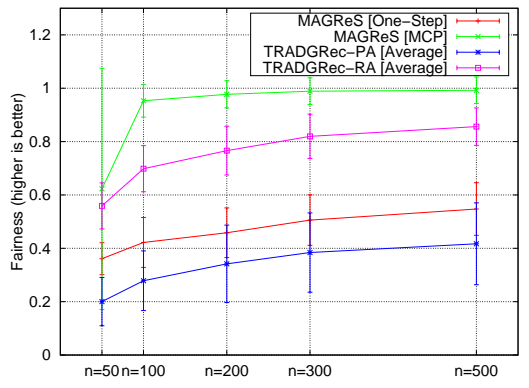

(c) Average fairness per approach and neighborhood size

Figure 3: Experimental results for the NN strategy.

area in which the places the user has rated and usual visits are) of each user, and then selects the neighbors of the target user among those within a certain radius (parameter), independently of the ratings they have given to the same POIs. In both strategies, we test with 5 neighborhood sizes (N): 50, 100, 200, 300 and 500. As for $N N U Z$, we use three different values for the radius parameter: 1,3 and $5 \mathrm{kms}$. More details of the configurations used for the different algorithms are provided in the project site ${ }^{5}$.

\subsection{Results}

Next, we summarize the main results obtained when comparing the recommendations generated by MAGReS, against those generated by TRADGRec-PA and TRADGRec-RA when using the two neighborhood selection strategies (NN and NNUZ). Space limitations preclude us from including all the performed experiments and results, but they are described in the project site.

\footnotetext{
${ }^{5}$ https://github.com/sschia/magres
} 
Figure 3 shows the results for the NN strategy. As it can be seen, independently of the size of the neighborhood (n), MAGReS [MCP] outperforms TRADGRec-PA [Average] and TRADGRec-RA [Average] in terms of GS (group satisfaction), as all the recommendations produced by the former had a higher GS than the recommendations generated by the latter two. Additionally, $M A$ GReS [One-Step] is only able to outperform TRADGRec-PA [Average], but it fails to do so when compared to MAGReS [MCP] and TRADGRec-RA [Average]. This can be explained by how the One-Step protocol works.

Regarding MSD and fairness, Tables $1 \mathrm{~b}$ and 1c show that MAGReS [MCP] outperforms all the other approaches. This indicates that MAGReS [MCP] recommendations not only have a higher group satisfaction (GS) score, but also satisfy the group members more uniformly (because of the lower MSD score) and ensure that a higher percentage of the group members will be satisfied with the recommendation (due to the higher fairness score).

For the NNUZ strategy, we obtained similar results as in the NN strategy, except when the zone radius $(z r)$ parameter was set to 1 (i.e. $1 \mathrm{~km}$ ). As shown in Table 1, MAGReS [MCP] outperformes all the other approaches for GS, MSD and Fairness for any neighborhood size, when $z r$ was set to 3 or 5 . When $z r$ was set to 1 , despite the neighborhood size $(n)$ chosen, we found similar trends as in $N N$ [n=50]: many group recommendations were empty, which lowered the averages and increased the deviations for all metrics (GS, MSD and Fairness).

\section{Conclusions}

In this paper, we analyzed and compared three approaches for POI group recommendation. Differently from works based on aggregation strategies, we propose a MAS that uses negotiation techniques to make group recommendations. Similar to our approach is the one presented in [13] that uses a MAS for generating group recommendations in the POI domain, but with some key differences: it requires "live user intervention" as candidate proposals are generated using a pool of items rated by all the users; the mediator relies on aggregation techniques to compute the group rating of the items; the mediator executes the protocol and generates the proposals; the agents can generate counter-offers and model users' behavior in conflicingt situations. We could not compare with it because it requires live user intervention.

The experiments showed two main findings: i) the use of negotiation instead of aggregation techniques can improve the quality of POI recommendations, not only increasing the level of satisfaction of the group but also satisfying group members in a more even way; ii) the information provided by the LBSN about geographical relationships among the users helps to improve the quality of recommendations (for small neighborhoods, e.g., up to 200 neighbors) while also making the neighbors selection process more efficient, as less user-user similarity computations are needed. Although we obtained satisfactory results, our experiments also had some limitations. Our current implementation relies on the users' utility/satisfaction function in the prediction made by the SURs. Along this line, increasing the quality of the predictions by using a different approach [2] could improve the recommendations. Regarding the role of the LBSN we believe that 


\begin{tabular}{|c|c|c|c|c|}
\hline Group Satisfaction & MAGReS [One-Step] & MAGReS [MCP] & TRADGRec-PA [Average] & TRADGRec-RA [Average] \\
\hline NNUZ $[\mathrm{n}=50, \mathrm{zr}=1]$ & $0.3642 \pm 0.0829$ & $0.4727 \pm 0.4159$ & $0.0914 \pm 0.1161$ & $0.5334 \pm 0.1294$ \\
\hline $\mathrm{NNUZ}[\mathrm{n}=50, \mathrm{zr}=3]$ & $0.3627 \pm 0.0781$ & $0.6548 \pm 0.3573$ & $0.1595 \pm 0.1043$ & $0.5453 \pm 0.1173$ \\
\hline NNUZ $[\mathrm{n}=50, \mathrm{zr}=5]$ & $0.3732 \pm 0.0827$ & $0.5946 \pm 0.3888$ & $0.1714 \pm 0.1078$ & $0.5686 \pm 0.0987$ \\
\hline NNUZ $[\mathrm{n}=100, \mathrm{zr}=1]$ & $0.3986 \pm 0.0895$ & $0.5075 \pm 0.4263$ & $0.1245 \pm 0.1509$ & $0.5745 \pm 0.1245$ \\
\hline NNUZ $[\mathrm{n}=100, \mathrm{zr}=3]$ & $0.4031 \pm 0.0923$ & $0.7572 \pm 0.3043$ & $0.2200 \pm 0.1411$ & $\frac{.0800 .1240}{0.6051 \pm 0.1137}$ \\
\hline NNUZ $[\mathrm{n}=100, \mathrm{zr}=5]$ & $0.4069 \pm 0.0751$ & $0.8380 \pm 0.1884$ & $0.2280 \pm 0.1300$ & $0.6369 \pm 0.1144$ \\
\hline NNUZ $[\mathrm{n}=200, \mathrm{zr}=1]$ & $0.4142 \pm 0.0847$ & & $0.1822 \pm 0.1962$ & $0.6164 \pm 0.1266$ \\
\hline NNUZ $[\mathrm{n}=200, \mathrm{zr}=3]$ & $0.4383 \pm 0.0899$ & $0.8173 \pm 0.2667$ & $0.2617 \pm 0.1792$ & $0.010738 \pm 0.1138$ \\
\hline NNUZ $[\mathrm{n}=200, \mathrm{zr}=5]$ & $0.4456 \pm 0.0924$ & $0.8680 \pm 0.1965$ & $0.2634 \pm 0.1584$ & $0.6882 \pm 0.0977$ \\
\hline NNUZ $[\mathrm{n}=300, \mathrm{zr}=1]$ & $0.4211 \pm 0.1026$ & $0.5554 \pm 0.4272$ & $0.1920 \pm 0.2008$ & $0.6363 \pm 0.1310$ \\
\hline NNUZ $[\mathrm{n}=300, \mathrm{zr}=3]$ & $0.4467 \pm 0.1029$ & $0.8124 \pm 0.2958$ & $0.2813 \pm 0.1902$ & $0.6957 \pm 0.1004$ \\
\hline NNUZ $[\mathrm{n}=300, \mathrm{zr}=5]$ & $0.4487 \pm 0.0932$ & $0.8787 \pm 0.1997$ & $0.2761 \pm 0.1837$ & $0.7064 \pm 0.1055$ \\
\hline NNUZ $[\mathrm{n}=500$ & $0.4423 \pm 0.1215$ & $0.5621 \pm 0.431$ & $0.1993 \pm 0.2086$ & $0.6353 \pm 0.1321$ \\
\hline NNUZ $[\mathrm{n}=500, \mathrm{zr}=3]$ & $0.4578 \pm 0.0924$ & $0.8373 \pm 0.2712$ & $0.2987 \pm 0.1921$ & $0.7110 \pm 0.0908$ \\
\hline NNUZ $[\mathrm{n}=500, \mathrm{zr}=5]$ & $0.4850 \pm 0.1022$ & $0.9088 \pm 0.1514$ & $0.2817 \pm 0.1720$ & $0.7248 \pm 0.1206$ \\
\hline
\end{tabular}

(a) Average GS per approach and neighborhood size

\begin{tabular}{|c|c|c|c|c|}
\hline $\begin{array}{l}\text { NNUZ }[\mathrm{n}=50, \mathbf{z r}=1] \\
\text { NNUZ }[\mathrm{n}=50, \mathbf{z r}=3] \\
\text { NNUZ }[\mathrm{n}=50, \mathbf{z r}=5]\end{array}$ & $\begin{array}{l}0.1600 \pm 0.0850 \\
0.1693 \pm 0.0665 \\
0.1610 \pm 0.0827\end{array}$ & $\begin{array}{l}0.0768 \pm 0.1384 \\
0.0499 \pm 0.0758 \\
0.0532 \pm 0.1023 \\
\end{array}$ & $\begin{array}{l}0.0752 \pm 0.0870 \\
0.1155 \pm 0.0878 \\
0.1314 \pm 0.0907\end{array}$ & $\begin{array}{l}0.2364 \pm 0.1118 \\
0.2147 \pm 0.0898 \\
0.1970 \pm 0.0939\end{array}$ \\
\hline $\begin{array}{l}\text { NNUZ }[\mathrm{n}=100, \mathrm{zr}=1] \\
\text { NNUZ }[\mathrm{n}=100, \mathrm{zr}=3] \\
\text { NNUZ }[\mathrm{n}=100, \mathrm{zr}=5]\end{array}$ & $\begin{array}{l}0.1863 \pm 0.0839 \\
0.1606 \pm 0.0724 \\
0.1360 \pm 0.0834\end{array}$ & $\begin{array}{l}\frac{0.0745 \pm 0.1528}{0.0435 \pm 0.0787} \\
\underline{0.0467 \pm 0.0829} \\
\end{array}$ & $\begin{array}{l}0.0914 \pm 0.0991 \\
0.1316 \pm 0.1906 \\
0.1326 \pm 0.0848\end{array}$ & $\begin{array}{l}0.2426 \pm 0.0963 \\
0.1906 \pm 0.1139 \\
0.1922 \pm 0.0950\end{array}$ \\
\hline $\begin{array}{l}\text { NNUZ }[\mathrm{n}=200, \mathrm{zr}=1] \\
\text { NNUZ }[\mathrm{n}=200, \mathrm{zr}=3] \\
\text { NNUZ }[\mathrm{n}=200, \mathrm{zr}=5]\end{array}$ & $\begin{array}{l}0.1882 \pm 0.0949 \\
0.1674 \pm 0.0767 \\
0.1654 \pm 0.0777\end{array}$ & $\begin{array}{l}\frac{0.0845 \pm 0.1571}{0.0517 \pm 0.0930} \\
\underline{0.0406 \pm 0.0829}\end{array}$ & $\begin{array}{l}0.1221 \pm 0.1232 \\
0.1484 \pm 0.1124 \\
0.1590 \pm 0.0092\end{array}$ & $\begin{array}{l}0.2275 \pm 0.1211 \\
0.1954 \pm 0.1008 \\
0.1749 \pm 0.1070\end{array}$ \\
\hline $\begin{array}{l}\text { NNUZ }[\mathrm{n}=300, \mathrm{zr}=1] \\
\text { NNUZ }[\mathrm{n}=300, \mathrm{zr}=3] \\
\text { NNUZ }[\mathrm{n}=300, \mathrm{zr}=5]\end{array}$ & $\begin{array}{l}0.1843 \pm 0.0939 \\
0.1639 \pm 0.0812 \\
0.1687 \pm 0.0778\end{array}$ & $\frac{0.0874 \pm 0.1605}{\frac{0.0411 \pm 0.08222}{0.0413 \pm 0.0831}}$ & $\begin{array}{l}0.1336 \pm 0.1339 \\
0.1428 \pm 0.0962 \\
0.1602 \pm 0.1119\end{array}$ & $\begin{array}{l}0.2217 \pm 0.1122 \\
0.1958 \pm 0.0975 \\
0.1902 \pm 0.0904\end{array}$ \\
\hline $\begin{array}{l}\text { NNUZ }[\mathrm{n}=500, \mathbf{z r}=1] \\
\text { NNUZ }[\mathrm{n}=500, \mathbf{z r}=3] \\
\text { NNUZ }[\mathrm{n}=500, \mathbf{z r}=5]\end{array}$ & $\begin{array}{l}0.2050 \pm 0.0887 \\
0.1747 \pm 0.0795 \\
0.1569 \pm 0.0763\end{array}$ & $\frac{0.0818 \pm 0.1559}{\frac{0.0489 \pm 0.0865}{0.0482 \pm 0.0934}}$ & $\begin{array}{l}0.1391 \pm 0.1393 \\
0.1606 \pm 0.0946 \\
0.1453 \pm 0.0960\end{array}$ & $\begin{array}{l}0.2134 \pm 0.1156 \\
0.1824 \pm 0.0966 \\
0.1783 \pm 0.0898\end{array}$ \\
\hline
\end{tabular}

(b) Average MSD per approach and neighborhood size

\begin{tabular}{|c|c|c|c|c|}
\hline Fairness & MAGReS [One-Step] & MAGReS [MCP] & DGRec-PA [Average] & TRADGRec-RA [Average] \\
\hline $\begin{array}{l}\text { NNUZ }[\mathrm{n}=50, \mathbf{z r}=1] \\
\text { NNUZ }[\mathrm{n}=50, \mathbf{z r}=3] \\
\text { NNUZ [n=50,zr=5] }\end{array}$ & $\begin{array}{l}0.3683 \pm 0.0844 \\
0.3654 \pm 0.0856 \\
0.4624 \pm 0.0867\end{array}$ & $\begin{array}{l}0.5212 \pm 0.4617 \\
0.7337 \pm 0.4046 \\
0.6502 \pm 0.4286\end{array}$ & $\begin{array}{l}0.0956 \pm 0.1242 \\
0.1586 \pm 0.1073 \\
0.1747 \pm 0.1139\end{array}$ & $\begin{array}{l}\frac{0.5424 \pm 0.1419}{0.5511 \pm 0.1319} \\
0.5749 \pm 0.1023\end{array}$ \\
\hline $\begin{array}{l}\text { NNUZ }[\mathrm{n}=100, \mathbf{z r}=1] \\
\text { NNUZ }[\mathrm{n}=100, \mathbf{z r}=3] \\
\text { NNUZ }[\mathrm{n}=100, \mathbf{z r}=5]\end{array}$ & $\begin{array}{l}0.4023 \pm 0.0934 \\
0.3997 \pm 0.1014 \\
0.4119 \pm 0.0831\end{array}$ & $\begin{array}{l}0.5485 \pm 0.4628 \\
0.8315 \pm 0.3355 \\
0.9130 \pm 0.2066 \\
\end{array}$ & $\begin{array}{l}0.1266 \pm 0.1555 \\
0.2292 \pm 0.1483 \\
0.2366 \pm 0.1392\end{array}$ & $\begin{array}{l}0.5808 \pm 0.1343 \\
0.6109 \pm 0.1298 \\
0.6509 \pm 0.1318\end{array}$ \\
\hline $\begin{array}{l}\text { NNUZ }[\mathrm{n}=200, \mathrm{zr}=1] \\
\text { NNUZ }[\mathrm{n}=200, \mathrm{zr}=3] \\
\text { NNUZ }[\mathrm{n}=200, \mathrm{zr}=5]\end{array}$ & $\begin{array}{l}0.4161 \pm 0.0898 \\
0.4378 \pm 0.0926 \\
0.4457 \pm 0.0992\end{array}$ & $\begin{array}{l}0.6125 \pm 0.4500 \\
0.8753 \pm 0.2887 \\
0.9263 \pm 0.2094 \\
\end{array}$ & $\begin{array}{l}0.1817 \pm 0.2042 \\
0.2610 \pm 0.1870 \\
0.2667 \pm 0.1662\end{array}$ & $\begin{array}{l}\frac{0.6265 \pm 0.1426}{0.6806 \pm 0.1250} \\
0.6999 \pm 0.1132\end{array}$ \\
\hline $\begin{array}{l}\text { NNUZ }[\mathrm{n}=300, \mathrm{zr}=1] \\
\text { NNUZ }[\mathrm{n}=300, \mathrm{zr}=3] \\
\text { NNUZ }[\mathrm{n}=300, \mathrm{zr}=5]\end{array}$ & $\begin{array}{l}0.4214 \pm 0.1065 \\
0.4431 \pm 0.1112 \\
0.4495 \pm 0.0987\end{array}$ & $\begin{array}{l}0.5890 \pm 0.4542 \\
0.8641 \pm 0.3139 \\
\underline{0.9310 \pm 0.2102} \\
\end{array}$ & $\begin{array}{l}0.1892 \pm 0.2077 \\
0.2851 \pm 0.1994 \\
0.2845 \pm 0.1946\end{array}$ & $\begin{array}{l}\frac{0.6464 \pm 0.1454}{0.7037 \pm 0.1097} \\
0.7217 \pm 0.1245\end{array}$ \\
\hline $\begin{array}{l}\text { NNUZ }[\mathrm{n}=500, \mathbf{z r}=1] \\
\text { NNUZ }[\mathrm{n}=500, \mathbf{z r}=3] \\
\text { NNUZ }[\mathrm{n}=500, \mathrm{zr}=5]\end{array}$ & $\begin{array}{l}0.4425 \pm 0.1260 \\
0.4493 \pm 0.0981 \\
0.4826 \pm 0.1084\end{array}$ & $\begin{array}{l}0.5831 \pm 0.4568 \\
0.8731 \pm 0.2968 \\
0.9503 \pm 0.1576\end{array}$ & $\begin{array}{l}0.1947 \pm 0.2107 \\
0.2920 \pm 0.2009 \\
0.2842 \pm 0.1769\end{array}$ & $\begin{array}{l}\frac{0.6429 \pm 0.1435}{0.7166 \pm 0.0997} \\
0.7336 \pm 0.1396\end{array}$ \\
\hline
\end{tabular}

(c) Average fairness per approach and neighborhood size

Table 1: Experimental results for the NNUZ strategy 
the LBSN information can help to further improve the recommendations if we include such information in the utility function used by the agents. For example, an agent might prefer POIs being closer to the movement area of her user.

Acknowledgements. We thank CONICET PIP Project 112-201501-00030, ANPCyT project PICT 2016-2973, C. Ríos and C. Villavicencio for their support and their work.

\section{References}

1. F. Ayala-Gómez, B. Daróczy, M. Mathioudakis, A. Benczúr, and A. Gionis. Where could we go?: Recommendations for groups in location-based social networks. In Proc. of the ACM on Web Science Conference (WebSci '17), pages 93-102, 2017.

2. L. Boratto, S. Carta, G. Fenu, F. Mulas, and P. Pilloni. Influence of rating prediction on group recommendation's accuracy. IEEE Int. Sys., 31(6):22-27, 2016.

3. U. Endriss. Monotonic concession protocols for multilateral negotiation. In Proc. 5th Int. Joint Conf. AAMAS '06, pages 392-399, 2006.

4. A. Felfernig, M. Stettinger, L. Boratto, and M. Tkalcic. Group Recommender Systems: An Introduction. Springer, 2018.

5. R. D. Gottapu and L. V. Sriram Monangi. Point-of-interest recommender system for social groups. Procedia Computer Science, 114(C):159-164, 2017.

6. G. Karypis. Evaluation of item-based top-n recommendation algorithms. In Proc. 10th Int. Conf. on Inf. and Knowl. Manag. (CIKM '01), pages 247-254, 2001.

7. T. N. Nguyen and F. Ricci. Dynamic elicitation of user preferences in a chat-based group recommender system. In Proc. SAC '17, pages 1685-1692, 2017.

8. S. Purushotham, C.-C. J. Kuo, J. Shahabdeen, and L. Nachman. Collaborative group-activity recommendation in location-based social networks. In Proc. 3rd ACM SIGSPATIAL Int. Workshop GeoCrowd '14, pages 8-15, 2014.

9. L. Ravi and S. Vairavasundaram. A collaborative location based travel recommendation system through enhanced rating prediction for the group of users. Computational Intelligence and Neuroscience, pages 7-, 2016.

10. F. Ricci, L. Rokach, and B. Shapira. Recommender Systems Handbook. Springer, 2nd edition, 2015.

11. C. Rios, S. Schiaffino, and D. Godoy. A study of neighbour selection strategies for POI recommendation in LBSNs. Journal of Inf. Sci., 44(6):802-817, 2018.

12. J. S. Rosenschein and G. Zlotkin. Rules of Encounter: Designing Conventions for Automated Negotiation Among Computers. MIT Press, 1994.

13. S. Rossi, C. Di Napoli, F. Barile, and L. Liguori. A multi-agent system for group decision support based on conflict resolution styles. In Conflict Resolution in Decision Making, pages 134-148. Springer, 2017.

14. C. Trattner, A. Said, L. Boratto, and A. Felfernig. Evaluating Group Recommender Systems, chapter 3, page 14. Springer, 2018.

15. C. Villavicencio, S. Schiaffino, J. A. Diaz-Pace, A. Monteserin, Y. Demazeau, and C. Adam. A mas approach for group recommendation based on negotiation techniques. In Proc. PAAMS 2016, volume 9662, pages 219-231. Springer, 2016.

16. Z. Yuan and C. Chen. Research on group POls recommendation fusion of users' gregariousness and activity in LBSN. In Proc. 2nd IEEE Int. Conf. on Cloud Computing and Big Data Analysis, pages 305-310, 2017.

17. F. L. B. Zeuthen. Problems of Monopoly and Economic Warfare. Routledge, 1930.

18. Q. Zhu, S. Wang, B. Cheng, Q. Sun, F. Yang, and R. N. Chang. Context-aware group recommendation for point-of-interests. IEEE Access, 6:12129-12144, 2018. 